\title{
Publisher Correction: Global characterization of T cells in non-small-cell lung cancer by single-cell sequencing
}

Xinyi Guo, Yuanyuan Zhang, Liangtao Zheng, Chunhong Zheng, Jintao Song, Qiming Zhang, Boxi Kang, Zhouzerui Liu, Liang Jin, Rui Xing, Ranran Gao, Lei Zhang, Minghui Dong, Xueda Hu, Xianwen Ren (D), Dennis Kirchhoff,

Helge Gottfried Roider, Tiansheng Yan and Zemin Zhang (D)

Correction to: Nature Medicine https://doi.org/10.1038/s41591-018-0045-3, published online 25 June 2018.

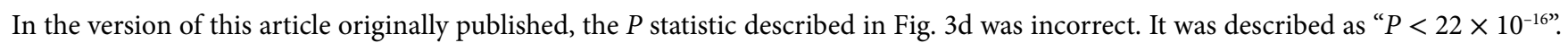
It should have been " $P<2.2 \times 10^{-16 "}$. Also, the "CD8 ${ }^{+}$Treg" label in Fig. $4 \mathrm{f}$ was incorrect. It should have been "CD4" Treg". The errors have been corrected in the HTML and PDF versions of this article.

Published online: 9 August 2018

https://doi.org/10.1038/s41591-018-0167-7

\section{Publisher Correction: Molecular phenomics and metagenomics of hepatic steatosis in non-diabetic obese women}

Lesley Hoyles (D), José-Manuel Fernández-Real, Massimo Federici (D), Matteo Serino (D), James Abbott (D), Julie Charpentier, Christophe Heymes, Jèssica Latorre Luque (D), Elodie Anthony, Richard H. Barton, Julien Chilloux (D), Antonis Myridakis (D), Laura Martinez-Gili D, José Maria Moreno-Navarrete, Fadila Benhamed, Vincent Azalbert, Vincent Blasco-Baque, Josep Puig, Gemma Xifra, Wifredo Ricart, Christopher Tomlinson (D), Mark Woodbridge (D), Marina Cardellini, Francesca Davato, Iris Cardolini, Ottavia Porzio, Paolo Gentileschi, Frédéric Lopez, Fabienne Foufelle, Sarah A. Butcher (D, Elaine Holmes, Jeremy K. Nicholson, Catherine Postic, Rémy Burcelin and Marc-Emmanuel Dumas (D)

Correction to: Nature Medicine https://doi.org/10.1038/s41591-018-0061-3, published online 25 June 2018.

In the version of this article originally published, the received date was missing. It should have been listed as 2 January 2018 . The error has been corrected in the HTML and PDF versions of this article.

Published online: 9 August 2018

https://doi.org/10.1038/s41591-018-0169-5

\section{Publisher Correction: Inactivating hepatic follistatin alleviates hyperglycemia}

Rongya Tao, Caixia Wang, Oliver Stöhr, Wei Qiu, Yue Hu, Ji Miao, X. Charlie Dong (D), Sining Leng, Margaret Stefater, Nicholas Stylopoulos, Lin Lin, Kyle D. Copps and Morris F. White

Correction to: Nature Medicine https://doi.org/10.1038/s41591-018-0048-0, published online 4 June 2018.

In the version of this article originally published, the $y$ axis labels in Fig. $4 \mathrm{~b}, \mathrm{~d}$ were incorrect. In Fig. $4 \mathrm{~b}$, the unit on the label was $\left(\mathrm{ng} \mathrm{mg}{ }^{-1}\right)$. This should have been (ng/ml). In Fig. 4d, the $y$ axis label was Serum Fst $\left(\mathrm{ng} \mathrm{ml}^{-1}\right)$. It should have been Serum insulin (ng/ml). The errors have been corrected in the HTML and PDF versions of this article. 